PROC. OF JSCE,

No. 247, MARCH 1976

\title{
SOME CHARACTERISTICS OF RAMP CONTROL ON URBAN EXPRESSWAY NETWORK
}

\author{
By Sho MYoIIN*, Hamao SAKAMOTO** and Shunsuke IWAMOTO***
}

\section{INTRODUCTION}

Several methods of ramp control have been proposed and devoted to traffic control system on urban expressway network. It is noticeable that ramp control in normal conditions is different in principle from that in emergency. In case of emergency when any incident is detected on expressway, the quickest possible recovery from, if any, traffic congestion should be aimed at by the control. On the other hand, in normal conditions that there exists no accidents on expressway, we set our sights on preventing any traffic stagnation due to excessive inflow of vehicles.

In general, ramp control in normal conditions requires any forecasting of traffic conditions on the whole network before and/or after its coming into operation. Some forecasting techniques have been developed. The following are some of them; preparing contour maps of vehicle concentration, speed, occupancy and so on ${ }^{1)}$, simulation run ${ }^{2)}$ or a direct forecasting of traffic volume in every section on expressway ${ }^{3}$.

In this paper we are concerned with LP (Linear Programming) control ${ }^{3)}$, which is one of the ramp control methods in normal conditions. The prerequisite to LP control is a direct forecasting mentioned above. It has been shown that the direct technique ensures a successful description of traffic volume on urban expressway4).

\section{SOME COMMENTS ON LP CONTROL}

LP control is stated as follows ${ }^{3}$; determine a vector $\boldsymbol{U}=\left(U_{1}, U_{2}, \cdots, U_{r}\right)$ so as to maximize the

* Dr. Eng., Assistant Professor, Department of Transportation Engineering, Kyoto University.

** Hanshin Expressway Public Corporation.

*** OMRON TATEISI Electronics Co. Central R \& D Lab, function $\sum U_{i}$ or $\sum U_{i} \bar{l}_{i}$ subject to $\boldsymbol{U} Q+\boldsymbol{\varepsilon} \leqq \boldsymbol{C}$ and $\mathbf{0} \leqq \boldsymbol{U} \leqq \boldsymbol{U}^{d}$, where $\boldsymbol{U}$ and $\boldsymbol{U}^{d}$ are vectors, whose entries express acceptable and forecasted inflow volume at entrance ramps, respectively, $\boldsymbol{C}$ is a capacity vector, $\varepsilon$ is an error vector defined by $\boldsymbol{Y}-\boldsymbol{U Q}$, where $\boldsymbol{Y}$ is an actual flow, and is introduced for eliminating prediction error ${ }^{4}, Q$ is called the unit inflow matrix and $\bar{l}_{i}$ is the average trip length of users from ramp $i$ on expressway network. The first inequality shows a constraint that traffic flow should not exceed the capacity in any section on network. The reason why we call this method LP control will be obvious from the foregoing.

In the foregoing, the objective function $\Sigma U_{i}$ means the total acceptable number of vehicles and $\sum U_{i} \bar{l}_{i}$ the total amount of trip length made by them on expressway. Some of the operational characteristics of LP control, using $\sum U_{i}$ as the objective function, were made clear by simulation $^{5)}$, according to which LP control is of the most successful applicability to steady flow, that is, to the flow that makes no transiently sharp change. At the same time, a problem was pointed out that LP control would lead to keeping toll gates on some specified ramps suppressed (closed) for a long period.

A measure was proposed to meet the situation by use of inequality $\boldsymbol{U}^{l} \leqq \boldsymbol{U} \leqq \boldsymbol{U}^{d}$ instead of the second one stated at the beginning. The vector $\boldsymbol{U}^{\boldsymbol{l}}$ represents the lowest limit of inflow and all of the factors are not zeros. It, however, is noticeable that the countermeasure gives rise to suplementary problem how $\boldsymbol{U}^{\imath}$ is to be determined.

LP control as stated firstly is accompanied by another problem of not a little importance that the waiting line of vehicles grows on approach to the ramp under steady suppression, and may disturb traffic flow on street. This problem may be relieved to some extent by the countermeasure mentioned above, because inequality $\boldsymbol{U}^{\imath} \leqq \boldsymbol{U} \leqq \boldsymbol{U}^{\boldsymbol{d}}$ has some effect of suppressing a rapid growth of 
waiting line even though it can not always prevent the line from growing to be obstacles to the street traffic. A more direct and effective measure to meet the case is to put some restraint on waiting line so that its tail end should not reach street. This is dealt with in the next section. In the following sections we call the LP control stated at the beginning the original LP control.

\section{MODIFICATION OF LP CONTROL}

By putting constraint upon queue length, the original LP control is modified as follows;

Determine an inflow vector $\boldsymbol{U}_{t}=\left(U_{1, t}, U_{2, t}, \cdots\right.$, $\left.U_{r, t}\right)$ so as to maximize $\sum_{i} U_{i, t}$ or $\sum_{i} U_{i, t} \vec{l}_{i}$ subject to

$$
\begin{array}{ll} 
& \boldsymbol{U}_{t} Q+\boldsymbol{\varepsilon} \leqq \boldsymbol{C} \\
& \boldsymbol{L}_{t}+\boldsymbol{U}_{\Delta t}^{d}-\boldsymbol{U}_{t} \leqq \boldsymbol{N} \\
\text { and } & \mathbf{0} \leqq \boldsymbol{U}_{t} \leqq \boldsymbol{L}_{t}+\boldsymbol{U}_{\Delta t}^{d}
\end{array}
$$

where $\boldsymbol{U}_{t}$ : inflow vector between time $t$ and $t+\Delta t$

$\boldsymbol{L}_{t}$ : queue length vector, whose factor represents the number of vehicles waiting at each ramp at time $t$,

$\boldsymbol{U}_{\Delta t}^{d}$ : arrival vector in which each entry expresses the number of arrivals forecasted to occur at each ramp in interval $\Delta t$ immediately after time $t$,

$\Delta t$ : time interval while a control is in operation,

$\boldsymbol{N}$ : allowable queue length vector, of which each entry represents the upper limit of the number of vehicles waiting at each ramp,

and the other notations have the same meanings as mentioned in the last section. The first and the second inequalities may be called capacity constraint and queue length constraints, respectively. Considering that inflow vector $\boldsymbol{U}_{t}$ is determined every time interval $\Delta t$, it is easy to understand that the queue length $\boldsymbol{L}_{t+\Delta t}=\boldsymbol{L}_{t}+$ $\boldsymbol{U}_{\Delta t}^{d}-\boldsymbol{U}_{t}$ is sure to be kept less than or equal to the upper limit $N$. This is the reason why the second inequality be called queue length constraint. Vector $\boldsymbol{N}$ has to be settled by taking both the geometric rampway conditions and traffic conditions on street into consideration.

The second and the last inequalities can be combined into the form

$$
\boldsymbol{L}_{t}+\boldsymbol{U}_{\Delta t}^{d}-\boldsymbol{N} \leqq \boldsymbol{U}_{t} \leqq \boldsymbol{L}_{t}+\boldsymbol{U}_{\Delta t}^{d}
$$

under nonnegative constraint $\mathbf{0} \leqq \boldsymbol{U}_{t}$. This expression will be used later in section 5 . It has been shown that operating time interval $\Delta t$ may as well be settled at shortest 5 minutes). In the above description of modified LP control, traffic capacity of booths is assumed to be infinite.

\section{SOME CHARACTERISTICS OF MODI- FIED LP CONTROL}

Some considerations are taken into as to the dynamic behaviors of modified LP control. For simplicity, arrival vector $\boldsymbol{U}_{\Delta t}^{d}$ is assumed to be time invariant and every factor of allowable queue length vector $\boldsymbol{N}$ to be large enough as compared with the corresponding one of $\boldsymbol{U}_{\Delta t}^{d}$. Time $t$ is represented discretely by $0,1,2, \cdots$, each of which is the time to perform a control operation. Starting at $t=0$ with the initial condition $\boldsymbol{L}_{0}=\mathbf{0}$ that there exists no waiting vehicle, control is performed successively as follows (Fig. 1);

(1) For the first step inflow vector $\boldsymbol{U}_{0}$ is given by the original LP control, that is subject only to $\boldsymbol{U}_{0} Q+\boldsymbol{\varepsilon} \leqq \boldsymbol{C}$ and $\mathbf{0} \leqq \boldsymbol{U}_{0} \leqq \boldsymbol{U}_{\Delta t}^{d} \quad\left(\boldsymbol{L}_{0}=\mathbf{0}\right)$ because queue length constraint does not act in fact by assumption that $\boldsymbol{U}_{\Delta t}^{d} \ll \boldsymbol{N}$. We have a set of entrance ramps under suppression, in which queue length grows to be

$$
\boldsymbol{L}_{1}=\boldsymbol{L}_{0}+\boldsymbol{U}_{\Delta t}^{d}-\boldsymbol{U}_{0}=\boldsymbol{U}_{\Delta t}^{d}-\boldsymbol{U}_{0}
$$

at the end of control interval $\Delta t$. At this stage, $\boldsymbol{L}_{1}$ is small enough as compared with $\boldsymbol{N}$.

(2) Control is performed successively in the same way as long as we have

$$
\boldsymbol{L}_{t+1}^{d}=\boldsymbol{L}_{t}+\boldsymbol{U}_{\Delta t}^{d} \leqq \boldsymbol{N}, \quad\left(\boldsymbol{U}_{\Delta t}^{d} \ll \boldsymbol{N} \text { by assumtion }\right)
$$

where $\boldsymbol{L}_{t+1}^{d}$ is an imaginary queue length vector that will be attainable at $t+1$ if every arrival in the interval is forced to wait, since queue length constraint does not act in fact while we have the above inequality. By assumption that $\boldsymbol{U}_{\Delta t}^{d}$ is time invariant, waiting lines grow by respective constant rates only in the fixed set of entrance ramps under suppression.

(3) New inflow vector $\boldsymbol{U}_{t}$ will be determined at time $t$ when we have firstly an imaginary queue length

$$
\boldsymbol{L}_{t+1}^{d}=\boldsymbol{L}_{t}+\boldsymbol{U}_{\Delta t}^{d}>\boldsymbol{N}
$$

at a single ramp or more included so far in the fixed set of suppressed ramps, for, otherwise, we should have an excess of queue length over the allowable. It is obvious that we have at time $t \boldsymbol{U}_{t}=\boldsymbol{L}_{t}+\boldsymbol{U}_{\Delta t}^{d}-\boldsymbol{N}$ and $\boldsymbol{L}_{t+1}=\boldsymbol{L}_{t}+\boldsymbol{U}_{\Delta t}^{d}-\boldsymbol{U}_{t}$ $=\boldsymbol{N}$ at those ramps where $\boldsymbol{L}_{t+1}^{d}>\boldsymbol{N}$. Inflow is fixed hereafter equal to arrival vector $\boldsymbol{U}_{\Delta t}^{d}$ as well as $\boldsymbol{L}_{t+1}$ to $\boldsymbol{N}$ at those ramps where $\boldsymbol{L}_{t+1}{ }^{d}>$ $\boldsymbol{N}$ because of queue length constraint, which means that those ramps are forced to allow just 
as large as the number of arrivals to flow in, keeping queue length fixed to each upper limit. Inversely, even at those ramps users can be released out of the waiting lines into expressway in time.

(4) Some time later, queue length on the other ramps reach each upper limit, and the rest of the ramps are newly placed under suppression in order. In course of time we will come to find no feasible inflow vector, which means that we can not go forward with LP control modified by queue length constraint.

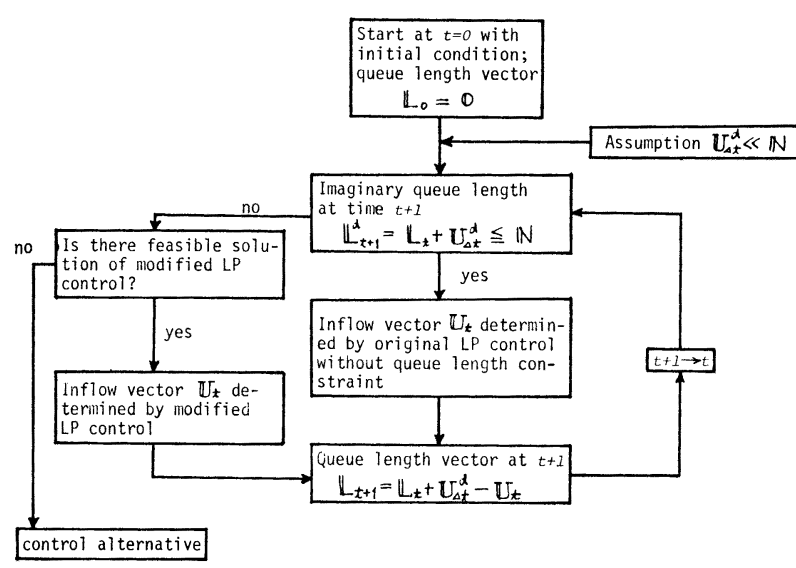

Fig. 1 Sequential step of Modified LP control.

The foregoing gives a simplified sequential expression of behaviors of the modified LP control. This control will be accompanied with the following problems;

(1) What is the desirable control alternative when the modified LP control gives feasible solution no longer.

(2) The merit of the modified LP control will be lost in some parts as the ramps under suppression continue to be large in number. Especially in case that comparatively large number of ramps are obliged to keep the waiting line length fixed to each upper limit, the optimization procedure may be little significant. Such a case is shown in the earlier part of the last step in the foregoing.

As for the first problem, there may be a scope left for further study on the following alternatives; a) the same control by relaxing capacity constraint (by shifting capacity vector on the right-hand side of capacity constraint) to such an extent that no heavy traffic congestion is caused on expressway, regardless of a little lowering of level of service, b) uniform control ${ }^{6}$, which gives inflow vector $\boldsymbol{U}_{t}$ by $\alpha \boldsymbol{U}_{\Delta t}^{d}$ where $\alpha$ is a scalar constant given by the ratio of an excess of traffic volume over the capacity to the forecasted volume in the section on expressway. Queue length constraint is naturally disregarded. It is pointed out that uniform control operates with just a small depreciation of utility of expressway when applied to flow nearly saturated all over the network, c) proportional control ${ }^{3)}$, which suppresses those entrance ramps alone that feed the bottleneck sections with traffic. This control may as well be adopted only when locally saturated and d) control by sequential ramp closing ${ }^{3}$, which shuts off the necessary and sufficient number of entrance ramps upstream nearest to each bottleneck section. Similarly to the second alternative, queue length constraint is cancelled in the last two.

The modified LP control will prove its worth especially in the following case; a) arrival intensity at each entrance ramp is rather light as compared with its waiting capacity, b) heavy arrivals have, if any, a rather short period of duration.

\section{NUMERICAL EXAMPLE}

Some characteristics of the modified LP control are illustrated by numerical examples together with the original one. As shown in Table 1 , four cases are dealt with by use of practical data. The area under study is shown in Fig. 2 which is a part bound for Osaka city along Osaka-Ikeda Routes on the Hanshin Expressway Network.

Table 1 A variety of LP control under study.

\begin{tabular}{|c|c|c|}
\hline Case No. & Objectiye function & Conditions \\
\hline 1 & $\begin{array}{l}\text { max. the sum total of } \\
\text { the number of vehicles } \\
\qquad \max \sum_{i} U_{i, t}\end{array}$ & $\begin{array}{l}\boldsymbol{U}_{t} Q+\boldsymbol{\varepsilon} \leqq \boldsymbol{C} \\
\boldsymbol{0} \leqq \boldsymbol{U}_{t} \leqq \boldsymbol{L}_{t}+\boldsymbol{U}_{\Delta t}^{d}\end{array}$ \\
\hline 2 & do. & $\begin{array}{c}\boldsymbol{U}_{t} Q+\boldsymbol{\varepsilon} \leqq \boldsymbol{C} \\
\boldsymbol{L}_{t}+\boldsymbol{U}_{\Delta t}^{d}-\boldsymbol{N} \leqq \boldsymbol{U}_{t} \leqq \boldsymbol{L}_{t}+\boldsymbol{U}_{\Delta t}^{d} \\
\quad \mathbf{0} \leqq \boldsymbol{U}_{t}\end{array}$ \\
\hline 3 & $\begin{array}{l}\max \text { the total amount } \\
\text { of vehicle-kilometers } \\
\quad \max \sum_{i} U_{i, t} \bar{l}_{i}\end{array}$ & $\begin{array}{l}\boldsymbol{U}_{t} Q+\boldsymbol{\varepsilon} \leqq \boldsymbol{C} \\
\boldsymbol{0} \leqq \boldsymbol{U}_{t} \leqq \boldsymbol{L}_{t}+\boldsymbol{U}_{\Delta t}^{d}\end{array}$ \\
\hline 4 & do. & $\begin{array}{c}\boldsymbol{U}_{t} Q+\boldsymbol{\varepsilon} \leqq \boldsymbol{C} \\
\boldsymbol{L}_{t}+\boldsymbol{U}_{\Delta t}^{d}-\boldsymbol{N} \leqq \boldsymbol{U}_{t} \leqq \boldsymbol{L}_{t}+\boldsymbol{U}_{\Delta t}^{d} \\
\mathbf{0} \leqq \boldsymbol{U}_{t}\end{array}$ \\
\hline
\end{tabular}

\section{(1) Input data}

Unit inflow matrix $Q$ is shown in Table 2 that is stored in the traffic control system of Hanshin 
Table 2 Unit inflow matrix.

\begin{tabular}{|c|c|c|c|c|c|c|c|c|}
\hline Name of entrance ramp & number & 103 & 105 & 107 & 109 & 111 & 113 & 117 \\
\hline Ikeda & (1) & 0.9999 & 0.9999 & 0.9781 & 0.9282 & 0.9282 & 0.9282 & 0.9282 \\
\hline Toyonaka-kita & (2) & 0 & 0.9999 & 0.9884 & 0.9555 & 0.9555 & 0.9555 & 0.9555 \\
\hline $\begin{array}{l}\text { Toyonaka-minami } \\
\text { (Shimada-guchi) }\end{array}$ & (3) & 0 & 0 & 0 & 0 & 0.9999 & 0.9999 & 0.9999 \\
\hline $\begin{array}{l}\text { Toyonaka-minami } \\
\text { (Meishin-guchi) }\end{array}$ & (4) & 0 & 0 & 0 & 0 & 0.9999 & 0.9999 & 0.9999 \\
\hline Kashima & (5) & 0 & 0 & 0 & 0 & 0 & 0.9999 & 0.9999 \\
\hline Tsukamoto & (6) & 0 & 0 & 0 & 0 & 0 & 0 & 0.9999 \\
\hline
\end{tabular}

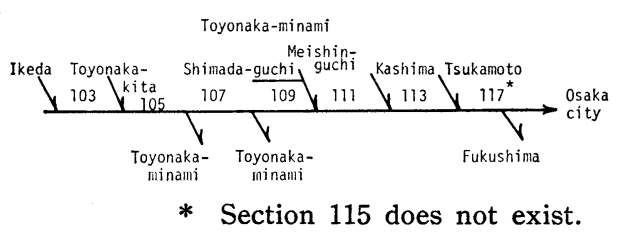

Fig. 2 Study area (Osaka-Ikeda route, bound for Osaka city).

Expressway as of the end of December, 1973.

Allowable queue length is shown in Table 3, which was given by actual surveying of the space available on each rampway.

Average trip length of users from each entrance ramp is also given in Table 3 , which is also used in the existing system.

The number of vehicle arrivals in the interval $\Delta t$ was observed at each ramp under study between 7:00 am. and 10:00 am., March 13, 1973, where $\Delta t$ was set 5 minutes. Fig. 3 shows the sequential data observed at Toyonaka-minami (Meishin-guchi) entrance ramp, one of the biggest ramps on the whole network.

Traffic capacity is set as 2,160 vehicles per hour per lane in every section under study, that is changed into 180 vehicles per 5 minutes per lane. By the way, we have two lanes in every study section and so we have 360 vehicles per five minutes as its capacity.

It is assumed that error vector $\varepsilon$ is equal to 0 .

\section{(2) Results of calculation}

Of the cases shown in Table 1, sequential solutions were obtained and compared each other as well as queue length, waiting time, traffic volume in a few sections and cumulative number of vehicles accepted to flow in.

In practice, an occurrence of traffic congestion due to an excessive inflows is observed in the study sections (most likely in section 117) regu-
Table 3 Allowable queue length and average trip length.

\begin{tabular}{l|c|c|c|c|c|c}
\hline Ramp no. & 1 & 2 & 3 & 4 & 5 & 6 \\
\hline $\begin{array}{l}\text { Allowable queue } \\
\text { length } N_{i} \\
\text { (vehicles) }\end{array}$ & 406 & 66 & 130 & 286 & 66 & 130 \\
\hline $\begin{array}{l}\text { Average trip } \\
\text { length } \bar{l}_{i}(\mathrm{~km})\end{array}$ & 16.22 & 16.01 & 13.99 & 12.55 & 12.03 & 10.82 \\
\hline
\end{tabular}

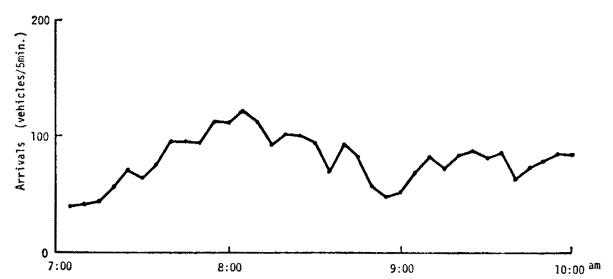

Fig. 3 The number of arrivals (Toyonakaminami, Meishin-guchi, ramp 4).

larly between 7:00 am. and 10:00 am. every weekday, and so this numerical examples may be regarded as nearly honest reproductions of the practical behaviors which would be brought about when each case of control was put into practice.

(a) Sequential solutions

The sequential solutions mean the time sequence of inflow vector. Fig. 4 shows schematically a change in performances of each case of control in time and space. In Fig. 4, the meanings of modes 1,2 and 3 are as follows;

mode 1: Vehicle is allowed to flow in without any suppression and further waiting. This mode is expressed by

$$
U_{i, t}=L_{i, t}+U_{i, \Delta t}^{d}
$$

where suffix $i$ is the ramp number. By the way, it is obvious that in this mode we have $U_{i, t}=U_{i, \Delta t}^{d}$ at ramp $i$ 
where no waiting line exists.

mode 2: Either no vehicle is allowed to flow in, or just an excess of the number of waiting vehicles over queue length limit is allowed to flow in. They are expressed either by

$$
U_{i, t}=0
$$

or by

$$
U_{i, t}=L_{i, t}+U_{i, \Delta t}^{d}-N_{i} \quad(>0)
$$

where suffix $i$ is the ramp number.

mode 3: The other solutions. This mode is expressed either by

$$
0<U_{i, t}<L_{i, t}+U_{i, \Delta t}^{d}
$$

or by

$$
\begin{aligned}
\max \left(0, L_{i, t}\right. & \left.+U_{i, \Delta t}^{d}-N_{i}\right) \\
& <U_{i, t}<L_{i, t}+U_{i, \Delta t}^{d}
\end{aligned}
$$

both of which mean that not all but none of the inflow demand $\left(L_{i, t}+\right.$ $\left.U_{i, \Delta t}^{d}\right)$ are allowed to flow in in the succeeding interval.

By the schematized presentation (Fig. 4) we have an outline of the control benavior in time and space.

(b) Queue length and waiting time

These two quantities are also measures of effectiveness of ramp control as well as the objective functions.

The quantities are evaluated with the cumulative amount of arrivals and inflows, as shown in

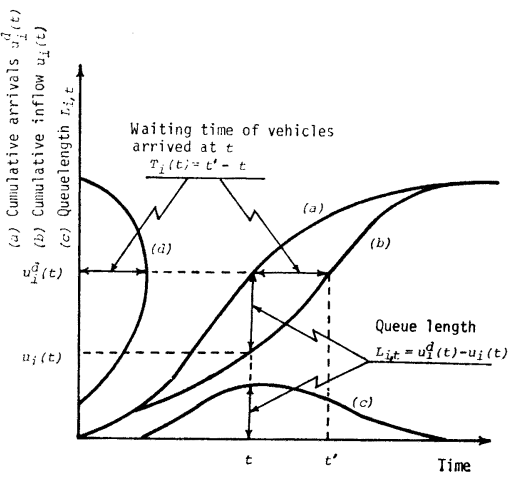

Fig. 5 Queue length and waiting time.

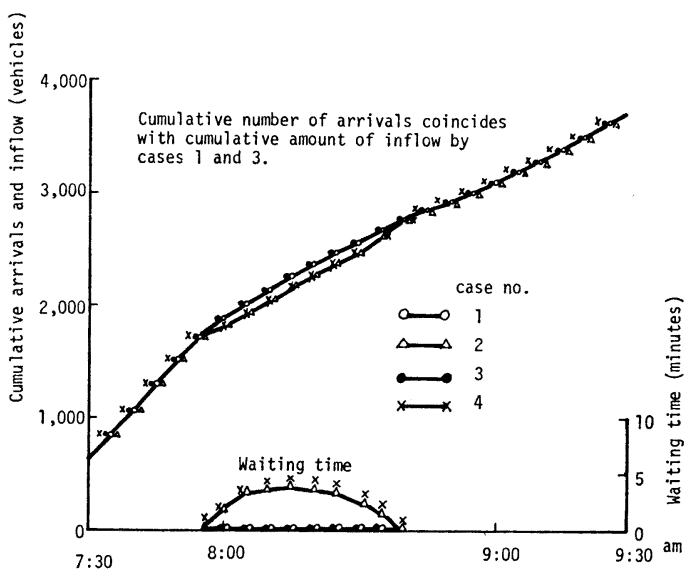

Fig. 6 Cumulative number of arrivals, cumulative amount of inflow and wait-

10:00 am ing time (Ikeda ramp, ramp 1).

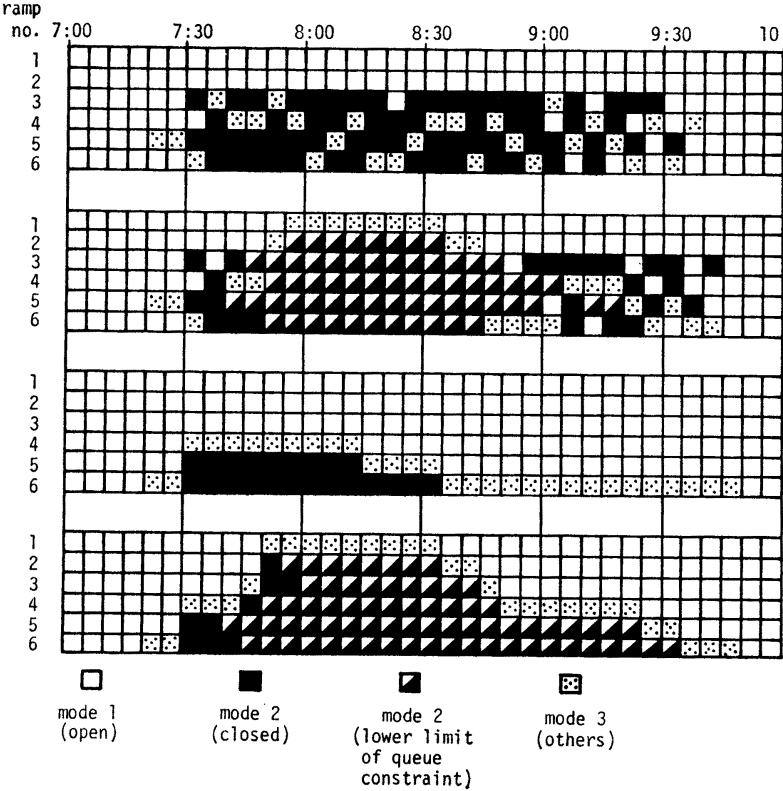

Fig. 4 Schema of the level of control.
Fig. 5.

Average number $L_{i}$ of waiting vehicles at ramp $i$ is given by

$$
\begin{aligned}
L_{i} & =\sum_{t=0}^{F} L_{i, t} / F \\
& =\sum_{t=0}^{F}\left\{u_{i}^{d}(t)-u_{i}(t)\right\} / F
\end{aligned}
$$

where

$L_{i, t}$ : the number of vehicles waiting at $\operatorname{ramp} i$ at time $t$,

$u_{i}^{d}(t)$ : the cumulative number of arrivals before time $t$,

$u_{i}(t)$ : the cumulative amount of inflow before time $t$,

and

$F$ : whole study time (by descrete expression).

Average waiting time is given by

$$
T_{i}=\sum_{i=0}^{F} U_{i, d t}^{d}(t) T_{i}(t) / u_{i}^{d}(F)
$$


where

$U_{i, \Delta t}^{d}(t)$ : the number of arrivals between $t$ and $t+\Delta t$, that was represented by $U_{i, \Delta t}^{d}$ in the earlier part,

$T_{i}(t)$ : waiting time of vehicle arriving at time $t$. We have $T_{i}(t)=t^{\prime}-t$, where $t^{\prime}$ is the time of flow-in when we have $u_{i}\left(t^{\prime}\right)=$ $u_{i}^{d}(t)$. Note that $t^{\prime}$ is uniquely determined because of the monotonic increase in cumulative number.

Tables 4 and 5 show the average number of waiting vehicles and the average waiting time, respectively.

Table 4 Average number of waiting vehicles (vehicles).

\begin{tabular}{c|c|c|c|c|c|c}
\hline Ramp no. & 1 & 2 & 3 & 4 & 5 & 6 \\
\hline Case no. & 0 & 0 & 59.9 & 119.4 & 72.8 & 67.8 \\
\hline 1 & 11.1 & 17.3 & 63.4 & 142.9 & 39.9 & 64.5 \\
\hline 2 & 0 & 0 & 0 & 41.0 & 54.9 & 238.3 \\
\hline 3 & 12.2 & 18.3 & 40.0 & 137.6 & 22.8 & 91.9 \\
\hline 4 &
\end{tabular}

Table 5 Average and the longest waiting time.

\begin{tabular}{|c|c|c|c|c|c|c|}
\hline & & & & \multicolumn{3}{|c|}{ (minutes) } \\
\hline Case no. & 1 & 2 & 3 & 4 & 5 & 6 \\
\hline 1 & 0 & 0 & $\begin{array}{r}11.59 \\
(33.0)\end{array}$ & $\begin{array}{r}8.12 \\
(26.0)\end{array}$ & $\begin{array}{l}10.87 \\
(36.0)\end{array}$ & $\begin{array}{r}7.31 \\
(21.0)\end{array}$ \\
\hline 2 & $\begin{array}{r}0.66 \\
(4.0)\end{array}$ & $\begin{array}{r}3.57 \\
(18.0)\end{array}$ & $\begin{array}{c}11.88 \\
(35.5)\end{array}$ & $\begin{array}{r}9.00 \\
(24.0)\end{array}$ & $\begin{array}{r}5.47 \\
(15.0)\end{array}$ & $\begin{array}{r}5.76 \\
(18.0)\end{array}$ \\
\hline 3 & 0 & 0 & 0 & $\begin{array}{r}2.66 \\
(13.5)\end{array}$ & $\begin{array}{r}7.05 \\
(42.0)\end{array}$ & $\begin{array}{r}22.90 \\
(66.0)\end{array}$ \\
\hline 4 & $\begin{array}{c}0.66 \\
(4.0)\end{array}$ & $\begin{array}{r}3.63 \\
(18.0)\end{array}$ & $\begin{array}{r}8.07 \\
(35.0)\end{array}$ & $\begin{array}{r}9.10 \\
(20.0)\end{array}$ & $\begin{array}{r}5.50 \\
(12.5)\end{array}$ & $\begin{array}{r}8.82 \\
(18.0)\end{array}$ \\
\hline
\end{tabular}

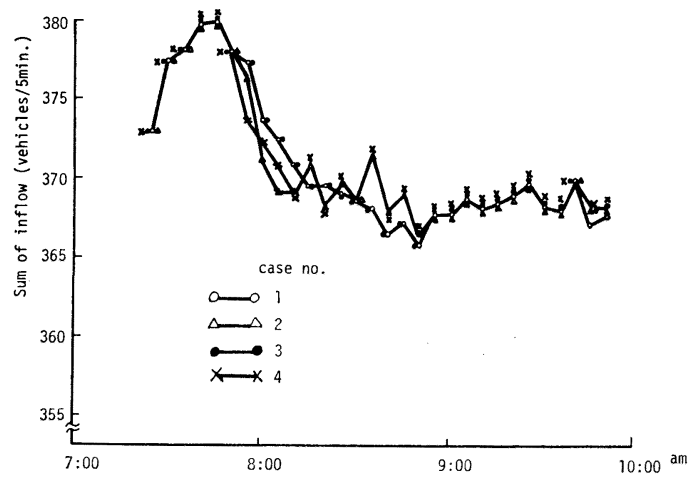

Fig. 7 Sequence of the sum total of inflow.

Fig. 8 shows a sequence of solutions (inflow) of the four cases, and Fig. 9 that of the number of waiting vehicles, both at Toyonaka-minami (Meishin-guchi) entrance ramp. The reason why this ramp is specified is that it seems to show some typical behaviors of control.

(e) Sequence of traffic volume in sections on expressway

They are shown in Figs. 10 and 11, which specify section number 111 and 117 respectively. Section 111 is picked out since it shows a wide range of fluctuation in traffic volume, which is one of the characteristics of each case of control. Note that section 111 is located immediately downstream to Toyonaka-minami (Meishin-guchi and Shimada-guchi) entrance ramp that has the largest number of vehicle arrivals of the ramps under study. Section 117 is the most downstream section in the study area, and it has during peak hour a regular excess of traffic volume over the capacity first of all sections under study. Fig. 11
Fig. 6 is a numerical example, corresponding to Fig. 5, that was obtained at Ikeda entrance ramp.

(c) The sum total of inflow

Fig. 7 shows a transition of the total number of vehicles accepted to flow in in each interval. Obviously the sum total of the number accepted throughout the whole time is equal to that of the arrivals in every case because we have a sharp decrease in arrival at most entrance ramps after peak hour.

(d) Sequences of inflow and queue length at Toyonakaminami entrance ramp

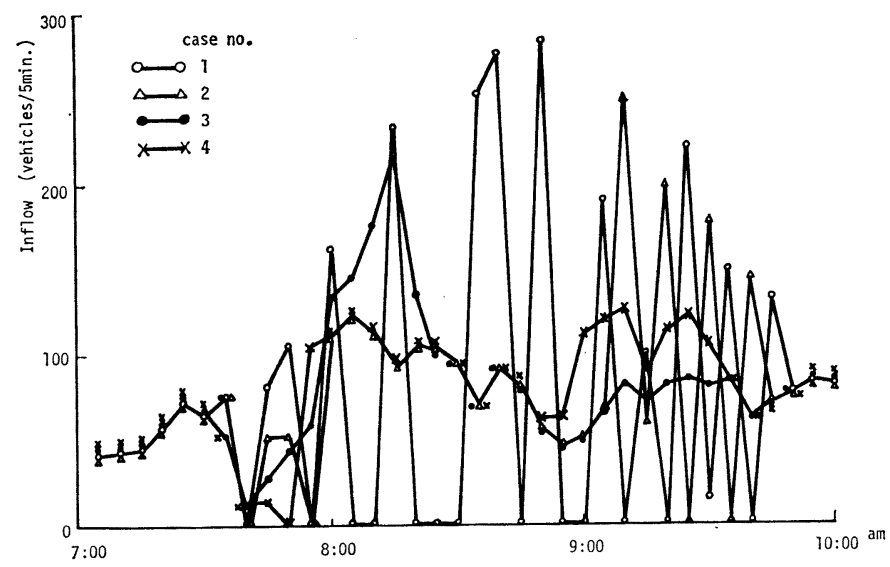

Fig. 8 Inflow at Toyonaka-minami (Meishin-guchi, ramp 4). 


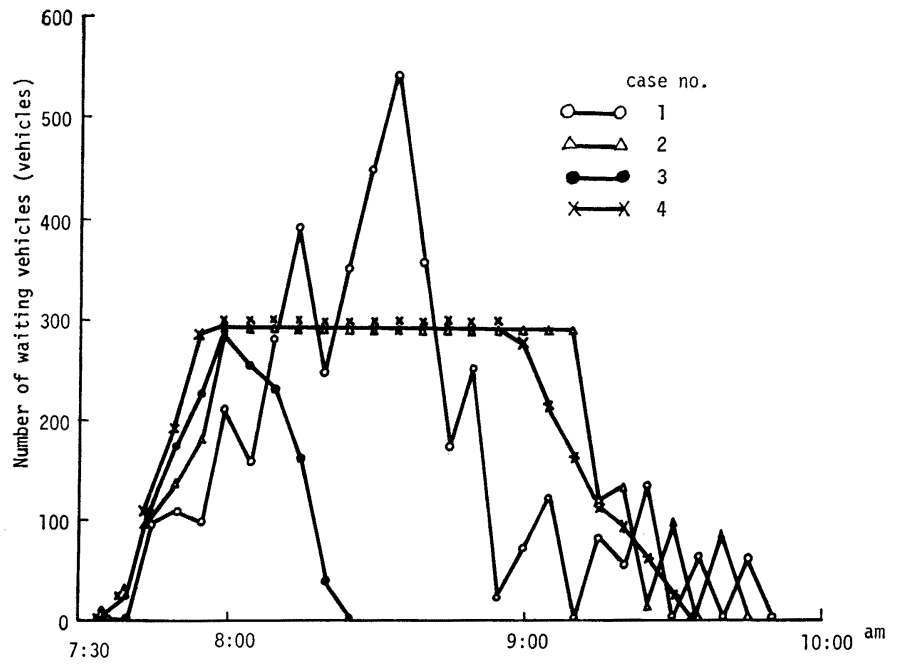

Fig. 9 The number of waiting vehicles (Toyonaka-minami,

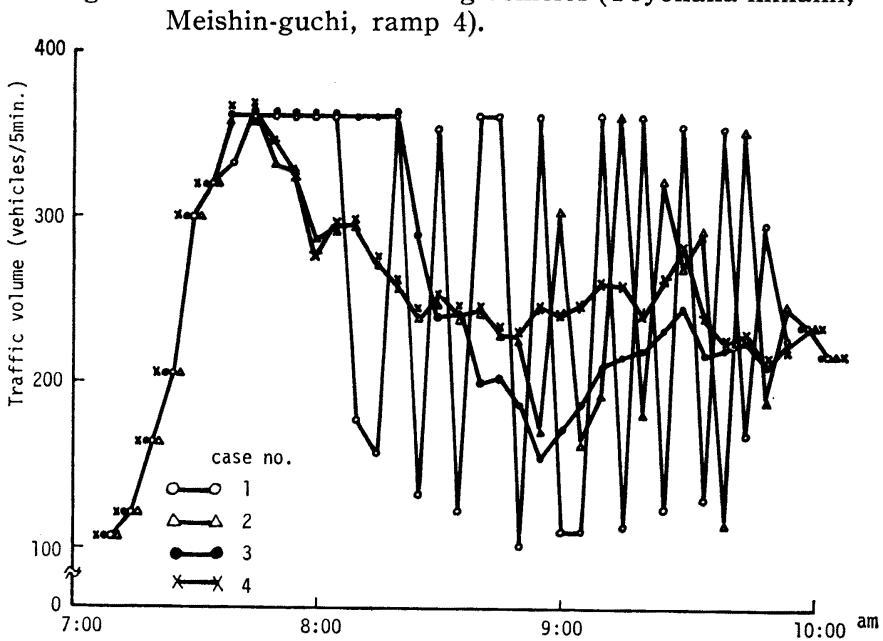

Fig. 10 Traffic volume in section 111.

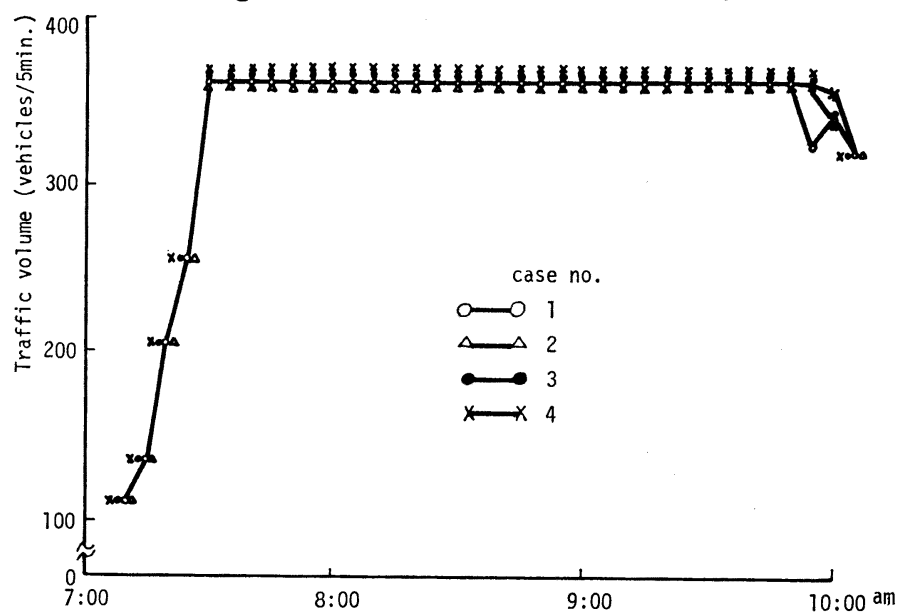

Fig. 11 Traffic volume in section 117 . shows that section 117 will be one of the bottlenecks throughout peak hour unless any ramp control is applied.

\section{(3) Detailed examinations of the four cases of control}

In this sub-section, we have some examinations of the performances of the four control cases separately, which will be summarized in the last section.

Case 1

(a) Fluctuations in inflow in space and time

Fig. 4 shows that entrance ramps are grouped into two sets; a set without suppression and another under suppression. The former includes ramps 1 and 2 and the latter ramps $3,4,5$ and 6 . Note that the former is located upstream to the latter and that the former's unit inflow factors to section 117 are smaller than the latter's (Table 2). Throughout almost whole peak hour we have a control pattern in the second set that a single ramp is under mode 3 while the other ramps under mode 2 .

It is remarkable that mode 3 shifts from one ramp to another in the second set. Remember that mode 3 is defined by $0<U_{i, t}<L_{i, t}$ $+U_{i, \Delta t}^{d}$ in this case and that to the second set should be assigned a volume given by

$\sum_{i \in s_{2}} U_{i, t}=\left(C_{117}-\sum_{i \in s_{1}} U_{i, \Delta t}^{d} Q_{i, 117}\right) / 0.9999$ where

$C_{117}$ : traffic capacity in section 117 ,

$Q_{i, 117}$ : unit inflow factor of ramp $i$ to section 117 ,

$s_{1}$ : the first set of ramps, which are under no suppression,

then, between 7:35 and 9:00 except a single interval, we find to be $0<\sum_{i \in s_{2}} U_{i, t}<L_{i, t}+U_{i, \Delta t}^{d}$ at least for those ramps $i$ of mode 3 in the second set $s_{2}$ of ramps that are under any suppression.

In this case, however, the opti- 
mum solution is essentially arbitrary in some set of solutions, since the ramps in the second set are all identical by unit inflow factor to section 117 (Table 2). That is, the optimum solution is arbitrary under the constraints

$$
\begin{aligned}
& \sum_{i \in s_{2}} U_{i, t}=\left(C_{117}-\sum_{i \in s_{1}} U_{i, t}^{d} Q_{i, 117}\right) / 0.9999 \\
& 0 \leqq U_{i, t} \leqq L_{i, t}+U_{i, \Delta t}^{d}
\end{aligned}
$$

The reason why a solution could be determined really in our example is that we stop further calculation when our algorithm has reached firstly an optimum point in the set of solutions above mentioned, and the reason why mode 3 shifts one ramp after another in the set $s_{2}$ is that the set $s_{1}$ fluctuates in arrival rate with time.

From viewpoint of level of service to users, it will never be preferable that the openning and shutting of the gates are repeated alternatively, moreover such an alternative operation of gates is troublesome in case of operation by manpower. A transition of suppression from one ramp to another, on the other hand, is desirable because of the possibility that queue may not grow to street.

(b) Queue length and waiting time

No waiting line exists in the set $s_{1}$, while queue grows in the set $s_{2}$ with fluctuation at each ramp till 8:30 when the cumulative amount of inflow is equal to the cumulative number of arrivals.

It is noticeable that we find a wide range of fluctuation in queue length (that is because of shifting of the modes).

Average waiting time in the set $s_{2}$ is between 7 and 12 minutes and the longest happens to be more than half an hour. The fluctuation in waiting time is over so wide range that it will be difficult for users to know in advance how long they have to wait.

(c) Traffic flow in sections on expressway

In section 117 traffic flow is kept constantly equal to the capacity by control, while section 111 has a very wide range of fluctuation according to the opening and shutting of gates at ramps immediately upstream to itself. A similar behavior was found in section 113.

(d) Sum total of the inflow volume

The behavior of fluctuation in the sum total of inflow is nearly similar to that in the number of arrivals at ramp 1. The reason is as follows; throughout peak hour, no suppression is forced in the set $s_{1}$ because we have in this example

$$
\Delta \equiv C_{117}-\sum_{i \in s_{1}} U_{i, \Delta t}^{d} Q_{i, 117}>0
$$

which should be allocated to the set $s_{2}$, and the sum of inflow is given by

$$
\sum_{i \in s_{1}} U_{i, \Delta t}^{d}+\Delta=C_{117}+\sum_{i \in s_{1}} U_{i, \Delta t}^{d}\left(1-Q_{i, 117}\right)
$$

that is dependent largely upon $U_{1, \Delta t}^{d}$ because $U_{1, \Delta t}^{d}$ is much larger than $U_{2, \Delta t}^{d}$ in our example. Case 2

(a) Fluctuations in inflow in space and time

Every ramp is suppressed by either mode 2 or 3. Especially between $8: 00$ and $8: 35$ all ramps but ramp 1 are filled up by waiting vehicles. Note that in this case there will be no feasible solution if ramp 1 happens to get filled up by waiting vehicles as well. Our example, however, happened to be equipped with enough waiting space at ramp 1 (Table 3). From Fig. 1 it is easy to understand that control modes in this case are similar to those in case 1 in the earlier and the later parts of peak period. Control modes shift in this case as well in case 1 in both parts of peak hour.

(b) Queue length and waiting time

Waiting line exists at every ramp (Table 4) and so does waiting time. A longest waiting time is shorter in this case than in case 1 as to ramp 4,5 and 6 , which should be because of queue length constraint (Fig. 9 for example). Each ramp, however, has in this case its proper fluctuation in waiting time in the middle of peak hour. The characteristic fluctuation is supposed to depend on [(allowable queue length)/(arrival rate minus inflow rate)]. For example, the reason why Toyonaka-minami (Shimada-guchi) entrance ramp (ramp 3) has the longest waiting time of all ramps is supposed that allowable queue is very long relative to the rate of arrival at ramp 3 while the one is not so long relative to the rate at the other ramps.

(c) Traffic flow in sections on expressway

It is noticeable that section 111 shows a wide range of fluctuation in traffic volume in the latter half of peak hour. A similar aspect was seen in section 113 immediately downstream to section 111.

(d) Sum total of the inflow volume

In the middle of peak period, the sum total fluctuates in the near reverse of the fluctuation in arrival rate over all the ramps but number 1. The reason is as follows; in the middle period queue length constraint forces ramp $i(i \neq 1)$ to flow in by each lowest limit

$$
\begin{aligned}
& \begin{array}{l}
\begin{array}{l}
U_{i, t} \\
=
\end{array} L_{i, t}+U_{i, \Delta t}^{d}-N_{i}, \quad i=2,3,4,5,6 \\
\quad=U_{i, \Delta t}^{d}
\end{array} \\
& \left(\because \quad L_{i, t}=N_{i} \text { in the middle period }\right)
\end{aligned}
$$

The rest of the capacity in section 117 is given 
by $C_{117}-\sum_{i=2}^{6} U_{i, \Delta t}^{d} Q_{i, 117}$, which is allocated to ramp 1 by increased volume of

$$
U_{1, t}=\left(C_{117}-\sum_{i=2}^{6} U_{i, \Delta t}^{d} Q_{i, 117}\right) / Q_{1,117}
$$

The sum total of inflow volume is given by

$$
\begin{aligned}
& \sum_{i=2}^{6} U_{i, t}+U_{1, t} \\
&=\left(\sum_{i=2}^{6} U_{i, \Delta t}^{d} Q_{1,117}+C_{117}-\sum_{i=2}^{6} U_{i, \Delta t}^{d} Q_{i, 117}\right) \\
& \times / Q_{1,117} \\
& \doteqdot\left(C_{117}-\left(1-Q_{1,117}\right) \sum_{i=2}^{6} U_{i, \Delta t}^{d}\right) / Q_{1,117} \\
& 1-Q_{1,117}>0
\end{aligned}
$$

where notations are similar to the ones before and $Q_{i, 117}$ may be put nearly equal to unity for $i=2,3, \cdots, 6$.

\section{Case 3}

(a) Fluctuations in inflow in space and time

From Fig. 4 it seems that the objective function to maximize the total amount of vehiclekilometers has such an effect as to keep control mode at each ramp stabilized in time. Needless to say, objective function $\sum_{i} U_{i, t} \bar{l}_{i}$ is easily changed into a form $\sum_{i} X_{i, t}$, by transformation that shifts unit inflow matrix by $\bar{l}_{i}$, which is similar to the form $\sum_{i} U_{i, t}$ in appearance.

It, however, should be empasized that we could have a unique solution in this case (in case 4 as well) because by transformation the unit inflow factors turn out to be different from each other in most practical cases. Obviously, the shorter average trip length a ramp has, the more likely the ramp is to be put into suppression. This is similar to the performance of Sequential Ramp Closing Control ${ }^{3}$, especially on the radial routes on expressway network.

(b) Queue length and waiting time

From Table 4 Tsukamoto entrance ramp.(ramp 6) has the largest average number of waiting vehicles, it has also the longest waiting time of all ramps both on the average and in the longest. Having the shortest trip length of all ramps, Tsukamoto ramp is most likely to be suppressed by modes 2 and 3 stationarily for a long period. Some of the users from Tsukamoto ramp happen to wait for one hour or more.

(c) Traffic flow in sections on expressway

Traffic flow in section 111 fluctuates not so sharply as seen in cases 1 and 2, but undergoes a rather stationary transition (Fig. 10).

(d) Sum total of the inflow volume
Transition of sum total is similar to that in case 1 (Fig. 7).

\section{Case 4}

(a) Fluctuations in inflow in space and time Control modes are distributed separatedly clearly over space and time (Fig. 4). This state of distribution seems to be a compound of those in cases 2 and 3 . Solutions are obtained uniquely as in case 3 .

(b) Queue length and waiting time

Tsukamoto ramp (ramp 6) has the shortest trip length together with the largest unit inflow factor of all rams, which is the reason why longest duration of the waiting line is found at this ramp. On the other hand, the longest waiting time is yielded not at this ramp but at Toyonaka-minami (Shimada-guchi, ramp 3), which is because of a dependence of waiting time on both allowable queue length and arrival rate as mentioned before.

(c) Traffic flow in sections on expressway

Traffic flow in section 111 fluctuates similarly to case 2 in the former half of peak hour, but it undergoes a transition between cases 2 and 3 in the latter half.

(d) Sum total of the inflow volume

Transition is nearly similar to in case 2 (Fig. 7).

\section{(4) Further discussions}

Some comments are given to objective function in this sub-section.

As mentioned previously, we happen to have an arbitrary solution in some set of solutions in case of adopting the total acceptable number of vehicles $\sum_{i} U_{i}$ (suffix $t$ is suppressed) as an objective function. In practical case an arbitrary solution will take place most likely on radial routes on expressway network, because unit inflow factors of some entrance ramps to a certain section are likely identical to each other on radial routes. In our example we realized an accidental shifting of control modes due to an arbitrariness of solution, which is likely to cause a sharp and wide range of fluctuations both in traffic flow in some sections on expressway and in level of service at some entrance ramps. This performance of control is never favorable to users.

A proposal has been made as follows ${ }^{7}$.

Combine objective function $\sum_{i} U_{i}$ with $\sum_{i} U_{i} \bar{l}_{i}$ in such a way that maximizes

$$
\sum_{i} U_{i} \bar{l}_{i}
$$

after having maximized 


$$
\sum_{i} U_{i}
$$

subject to

$$
\boldsymbol{U} Q+\boldsymbol{\varepsilon} \leqq \boldsymbol{C} \text { and } \mathbf{0} \leqq \boldsymbol{U} \leqq \boldsymbol{L}+\boldsymbol{U}^{d}
$$

or to

$$
\begin{aligned}
& \boldsymbol{U} Q+\boldsymbol{\varepsilon} \leqq \boldsymbol{C}, \quad \boldsymbol{L}+\boldsymbol{U}^{d}-\boldsymbol{N} \leqq \boldsymbol{U} \leqq \boldsymbol{L}+\boldsymbol{U}^{d} \\
& \text { and } \quad \boldsymbol{U} \geqq \mathbf{0}
\end{aligned}
$$

(suffix $t$ and $\Delta t$ are neglected).

A rather detailed description is given in the following.

Suppose that we have, after control that maximizes $\sum_{i} U_{i}$ subject to the first constraints, a set $J$ of such sections on expressway that, putting error term equal to zero,

$$
\sum_{i} U_{i} Q_{i j}=C_{j}, \quad j \in J=\left\{j_{1}, j_{2}, \cdots, j_{n}\right\}
$$

where the set $\left\{U_{i}\right\}$ is the one of the solutions, obtained not always uniquely by the first control.

A set $s_{j}$ is defined, if any, of two or more entrance ramps with a non-zero unit inflow factor $Q_{j}$, that is,

$$
\begin{aligned}
& Q_{i j} \equiv Q_{j}(\neq 0), \quad j \in J \\
& i \in s_{j}=\left\{i_{1}, i_{2}, \cdots, i_{n_{j}}\right\}, \quad n_{j} \geqq 2
\end{aligned}
$$

Concerning section $j$ alone for the present, we have a nonunique set $\left\{U_{i}^{\prime}\right\}, i \in s_{j}$, of solutions under the following constraints

$$
\begin{aligned}
& 0 \leqq U_{i^{\prime}} \leqq L_{i}+U_{i}{ }^{d}, \quad i \in s_{j} \\
& \sum_{i \in s_{j}} U_{i^{\prime}}=\sum_{i \in s_{j}} U_{i}=\Delta_{j} / Q_{j} \\
& \Delta_{j}=C_{j}-\sum_{i \notin s_{j}} U_{i} Q_{i j}
\end{aligned}
$$

if and only if

$$
0<\sum_{i \in s_{j}} U_{i}<\sum_{i \in s_{j}}\left(L_{i}+U_{i}^{d}\right)
$$

Note that we have a unique set $\left\{U_{i}\right\}, i \in s_{j}$, in case either $\sum_{i \in s_{j}} U_{i}=0$ or $\sum_{i \in s_{j}} U_{i}=\sum_{i \in s_{j}}\left(L_{i}+U_{i}^{d}\right)$ since we have a unique set $\{0\}$ or $\left\{L_{i}+U_{i}^{d}\right\}, i \in s_{j}$, respectively.

Our problem is now to determine $\left\{U_{i}{ }^{\prime}\right\}, i \in s_{j}$, so as to maximize the objective function $\sum_{i \in s_{j}} U_{i}^{\prime} \bar{l}_{i}$ subject to $0 \leqq U_{i}{ }^{\prime} \leqq L_{i}+U_{i}^{d}, \quad i \in s_{j}$, and $\sum_{i \in s_{j}} U_{i^{\prime}}=$ $\sum_{i \in s_{j}} U_{i}$. Obviously the unique solution is determined only by ranging $\left(L_{i}+U_{i}^{d}\right), i \in s_{j}$ by trip length when $\bar{l}_{i} \neq \bar{l}_{h}(i \neq h)$.

Similarly considering all possible sections, we have only to solve the problem that maximizes

$$
\sum_{i \in \bigcup_{j \in J} s_{j}} U_{i} \bar{l}_{i}
$$

under the following constraints

$$
0 \leqq U_{i^{\prime}} \leqq L_{i}+U_{i}^{d}, \quad i \in \underset{j \in J}{\cup} s_{j}
$$

$$
\sum_{i \in s_{j}} U_{i^{\prime}}=\sum_{i \in s_{j}} U_{i}=\Delta_{j} / Q_{j}, \quad j \in J
$$

in which

$$
0<\sum_{i \in s_{j}} U_{i}<\sum_{i \in s_{j}}\left(L_{i}+U_{\imath}^{d}\right), \quad j \in J
$$

The unique solutions are obtained similarly when $\bar{l}_{i} \neq \bar{l}_{h}(i \neq h)$ as is the practical case.

Illustration is made by use of the foregoing numerical example without queue length constraint, concerning section 117 alone.

Nearly between $7: 30$ and $9: 30$ we had a set $\{U\}$ of solutions that

$$
\begin{aligned}
& \sum U_{i} Q_{i j}=C_{j}, \quad j=117 \\
& 0<\sum_{i \in s_{2}} U_{i}<\sum_{i \in s_{2}}\left(L_{i}+U_{i}^{d}\right)
\end{aligned}
$$

where $s_{2}$ is the set of rams $3,4,5$ and 6 which have an identical unit inflow factor to the section 117, that is, $Q_{i j}=Q_{j}=0.9999\left(i \in s_{2}\right)$ (Table 2 and case 1 in Fig. 4).

Since $\bar{l}_{3}>\bar{l}_{4}>\bar{l}_{5}>\bar{l}_{6}, \quad \max _{i \in s_{2}} U_{i}^{\prime} \bar{l}_{i}$ under constraints of

$$
\begin{aligned}
& 0 \leqq U_{i}{ }^{\prime} \leqq L_{i}+U_{i}^{d}, \quad i \in s_{2} \\
& \sum_{i \in s_{2}} U_{i}{ }^{\prime}=\sum_{i \in s_{2}} U_{i}=\Delta_{j} / 0.9999 \\
& \Delta_{j}=C_{j}-\sum_{i=1,2} U_{i} Q_{i j}, \quad j=117
\end{aligned}
$$

is given by

(1) $U_{3}{ }^{\prime}=\sum_{i \in s_{2}} U_{i}$ and $U_{4}^{\prime}=U_{5}{ }^{\prime}=U_{6}{ }^{\prime}=0$ when $\sum_{i \in s_{2}} U_{i} \leqq L_{3}+U_{3}^{d}$

(2) $U_{3}{ }^{\prime}=L_{3}+U_{3}^{d}, \quad U_{4}{ }^{\prime}=\sum_{i \in s_{2}} U_{i}-U_{3}{ }^{\prime}$ and $U_{5^{\prime}}=$ $U_{6^{\prime}}=0$ when $L_{3}+U_{3}^{d}<\sum_{i \in s_{2}} U_{i} \leqq \sum_{i=3,4}\left(L_{i}+U_{i}^{d}\right)$

(3) $\quad U_{3}^{\prime}=L_{3}+U_{3}^{d}, \quad U_{4}^{\prime}=L_{4}+U_{4}^{d}, \quad U_{5}^{\prime}=\sum_{i \in s_{2}} U_{i}-$ $\left(U_{3}{ }^{\prime}+U_{4}{ }^{\prime}\right)$ and $U_{6}{ }^{\prime}=0$ when $\sum_{i=3,4}\left(L_{i}+U_{i}^{d}\right)$ $<\sum_{i \in s_{2}} U_{i} \leqq \sum_{i=3}^{5}\left(L_{i}+U_{i}^{i}\right)$

and so on.

\section{CONCLUSIONS}

Four cases of ramp control methods were examined and compared each other by numerical example. Some characteristics of their dynamic behaviors are summarized in the following.

(1) Case 4 is the most desirable control of the four cases since it may cause the least fluctuations both in traffic flow in the sections immediately downstream to some controlled entrance ramps and in the waiting line length at some controlled ramps, and since it would also give the least fluctuation in the level of service to users especially at big entrance ramp. 
(2) Objective function $\Sigma U_{i} \bar{l}_{i}$ has such an effect to give a higher priority of inflow to the ramp of longer trip length. Accordingly Case 3 would give a set of controlled ramps similar to sequential ramp closing control, especially on a radial route, since both would suppress those ramps located nearer upstream to the section where traffic volume is forecasted to exceed the capacity. A nearly similar behavior takes place in case 1 as well.

(3) In practice the original control, that maximizes the total acceptable number of vehicles, does not always put a fixed set of ramps steadily under certain modes of control. Both a fluctuation in the rate of arrival at each ramp and the characteristics of our algorithm used for calculation are the sources of transition from a certain set to another.

(4) LP control with objective function $\sum U_{i} \bar{l}_{i}$ is sure to settle a unique set of solutions since average trip length are seldom identical to each other.

(5) LP control modified by queue length constraint can achieve not only optimum operation of urban expressway network but also undisturbed traffic flow on the street near the entrance to expressway. We, however, have to pay attention to the fact that the modified LP control is depreciated in the cost-performance as compared with the original one, since the calculating cost of the modified control increases rapidly with an increase in the number of queue constraints added, while the performance is decreased by an increase in the number of entrance ramps where queue length reaches the upper limit.

(6) From the fact that it took a little less than one minute to solve our case study, the modified LP control is supposed to be applicable to an on-line-real-time control system of a rather small network (for example, a single radial way of usual length). In applying to a larger network it will be required to devide the network into some appropriate sub-networks, which is a problem left for further study.

(7) A ramp control with waiting time constraint is worth studying since the most users of urban expressway are sensitive to the whole travelling time on expressway, especially on toll expressway.

\section{ACKNOWLEDGEMENT}

The Research Committee for Traffic Control System of Hanshin Expressway, organized in 1973 by the Japan Society of Traffic Engineers, has been of valuable assistance to the authors because of the fruitful discussions in its meeting. The authors should like to express their gratitude to the leader of the Committee, Professor E. Kometani, who had a great influence on their study. They also express their thanks to the vice-leader, Professor T. Sasaki, for his many suggestive and helpful advices.

\section{REFERENCES}

1) Makigami, Y., L. Woodie and A. D. May: Bay Area Freeway Operations Study-Final Report, Analytic Techniques for Evaluating Freeway Improvements, Part I of III, ITTE Special Report, Aug. 1970.

Allen, B. L. and A. D. May: the same as above, Part II of III.

2) Nabeshima, Y.: Simulation Technique for Short Term Prediction of Traffic Condition, Research Materials of Japan Society for Simulation Technology, Vol. I-No. 2, Dec. 1973 (in Japanese).

3) Sasaki, T. and Myojin, S.: Theory of Inflow Control on an Urban Expressway System, Trans. of JSCE, No. 160, Dec. 1968.

4) Kometani, E. and Myojin, S.: A Short Term Forecasting of Traffic Volume on Urban Expressway Network, Traffic Engineering, Vol. 8, No. 5, Sept. 1973 (in Japanese).

5) Express Highway Research Foundation of Japan: Report of Study on Technical Research for Traffic Control on Hanshin Expressway, Special Report, March 1969 (in Japanese).

6) Sasaki, T. and Myojin, S.: Theory of Ramp Control on Urban Expressway Network, Traffic Engineering, Vol. 3, No. 3, May 1968 (in Japanese).

7) The idea was suggested originally by Professor T. Sasaki, who was a vice-leader of The Research Committee for Traffic Control System of Hanshin Expressway organized in 1973 by the Japan Society of Traffic Engineers.

(Received March 27, 1975) 